\title{
Raith - Electron Beam Lithography for Research
}

\author{
M. KIRCHNER AND M. KAHL \\ Raith GmbH, Konrad-Adenauer-Allee 8, 44263 Dortmund, Germany*
}

Electron beam lithography is one of the enabling methods in advanced nanoresearch. Electron beam lithography is a maskless lithography technique which is therefore very flexible. Electron beam lithography provides very high resolution. This is the main reason of its wide use in nanoresearch [1]. Electron beam lithography tools are in use in all engineering and basic science disciplines. Raith manufactures a variety of electron and ion beam lithography systems for research and development applications designed to meet the needs of researchers, designers, and engineers in both university and industry environment. Furthermore, Raith develops ultrahigh precision stages and navigation packages for failure analysis applications.

\section{Electron beam lithography tool components}

An electron beam lithography (EBL) tool consists of several components [2]. The main components are: the electron source, the electron optics, the deflection unit, the pattern generator, the sample stage. With the exception of the pattern generator these components are in principle available in a scanning electron microscope (SEM). Consequently, a SEM can be upgraded to a lithography tool. The user defines arbitrary patterns using CAD software and the pattern generator converts the CAD design into electronic commands to position the electron beam. SEM conversion solutions are in wide use. Better specifications are achieved with dedicated tools. This pays off especially for the applications discussed below (overlay, photonic crystals, and waveguides).

\section{The EBL process}

Samples for EBL (e.g. silicon, all type of semiconductors and metals, glass) are coated with a thin layer of resist which becomes either soluble (positive resist) or insoluble (negative resist) by the exposure with the electron beam. After developing, the temporary resist pattern is transferred into the substrate, typically either by etching or lift-off technique. The final result is a metal image or an etched image of the preceding CAD design work. In principle, any pattern is possible to be done,

* e-mail: www.raith.com; e-mail: sales@raith.de which gives the researcher the necessary flexibility [2]. In addition to $\mathrm{CAD}$ design data, other data like greyscale bitmaps can be also used for exposure. In the latter case $3 \mathrm{D}$ structures are the direct outcome of one exposure and one development step $[3,4]$.

\section{Achievable resolution}

One of the most important specifications of an EBL system is the minimum feature size, which is in the range of $10 \mathrm{~nm}$ of up-to-date systems [5]. Achievable minimum structure sizes are even below. For example, with the Raith $150^{\text {TWO }}$ EBL tool a line width of $5 \mathrm{~nm}$ has been obtained [6, 7].

\section{Overlay and mark finding (contacting nanowires)}

In multilayer designs - in which (at least) two layers have to match perfectly - good mark recognition is necessary to achieve good overlay accuracy. A special example of overlay technique is the contacting of nanowires to make 4 point conductivity measurements. There are two techniques to fabricate the contacts. The first one is EBL followed by lift-off. The second one isdirect deposition of the metal layer by electron beam induced de-

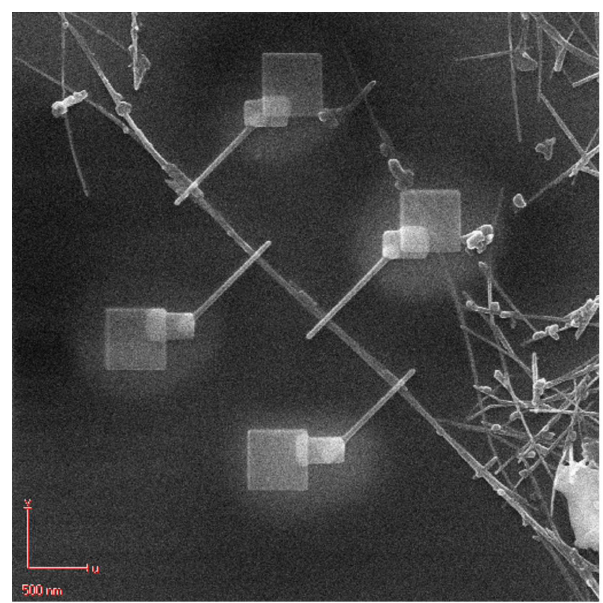

Fig. 1. "Wiring" of CNTs on $\mathrm{SiO}_{2}$-sample. S. Bauerdick, Raith GmbH. 
position (EBID, see Fig. 1) [8]. In each case the exact position of the nanowire relative to alignment marks has to be determined. This could challenge the imaging capabilities of the EBL tool, because some types of nanowires are extremely difficult to image.

The position data are the base of the CAD design of the contacting electrodes.

In the exposure step (after loading the sample again) a high precision sample stage helps to get back to a system of global and local markers. Dedicated EBL tools use laser interferometer controlled stages to get back to the marker system with higher precision. (Consequently the scan area for mark detection is considerably reduced and less sample area is wasted for mark detection.)

\section{Photonic crystals and proximity effect}

Photonic crystal devices are promising candidates for future generations of optical communication devices. However, their performance depends heavily on the correct placement, size, and shape of small circular structures. Therefore it is not enough to expose the desired
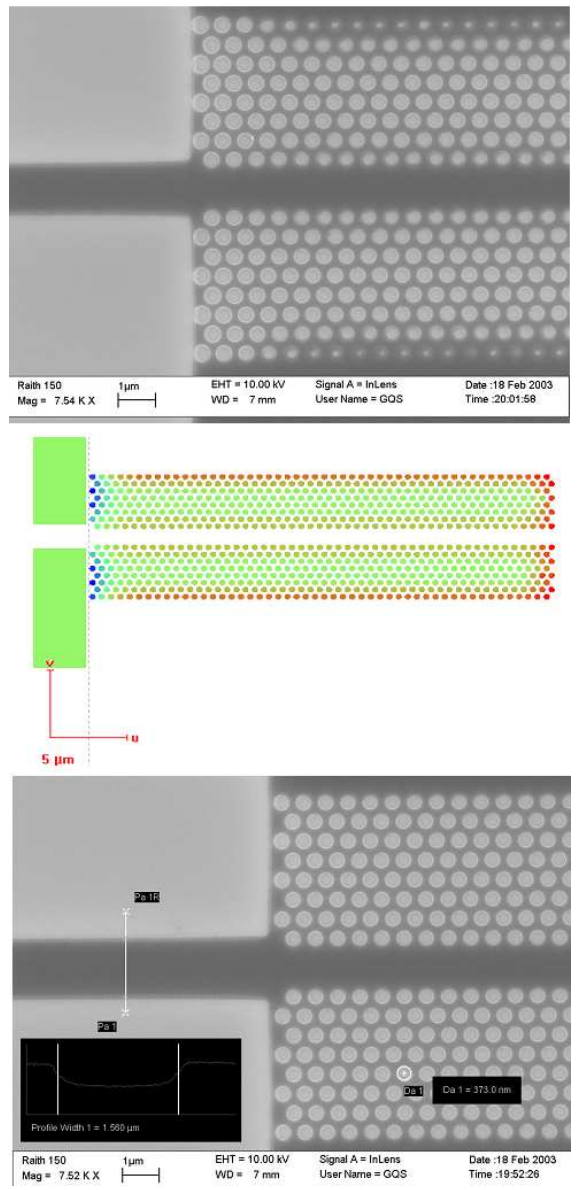

Fig. 2. Photonic crystal structure without (top) and with (bottom) PEC. PEC is achieved with dose assignment (middle). M. Kahl, Raith GmbH. layout with a constant dose distribution, which would result in a non-satisfactory pattern as shown in Fig. 2. The reason is the nature of the exposure process. Electrons are subject of forward and backward scattering in resist and substrate. The electron scattering results in additional exposure events at points adjacent to the point of impingement. In other words: the exposure of a feature depends on the proximity of this feature. However, for layouts that are sensitive to this effect a proximity effect correction (PEC) can be applied [9, 10]. In this case different parts of the design are assigned to individual doses (shown by false colours in the middle image of Fig. 2). Correct dose assignment leads to the satisfactory result as shown in the bottom image.

\section{Stitching and fixed beam moving stage (writing waveguides)}

The scan range of the electron beam (the write field) in any EBL tool is limited. To expose larger areas with several square millimetres or centimetres, several exposure fields have to be stitched together. Even with high precision stages there is a typical residualstitching error in

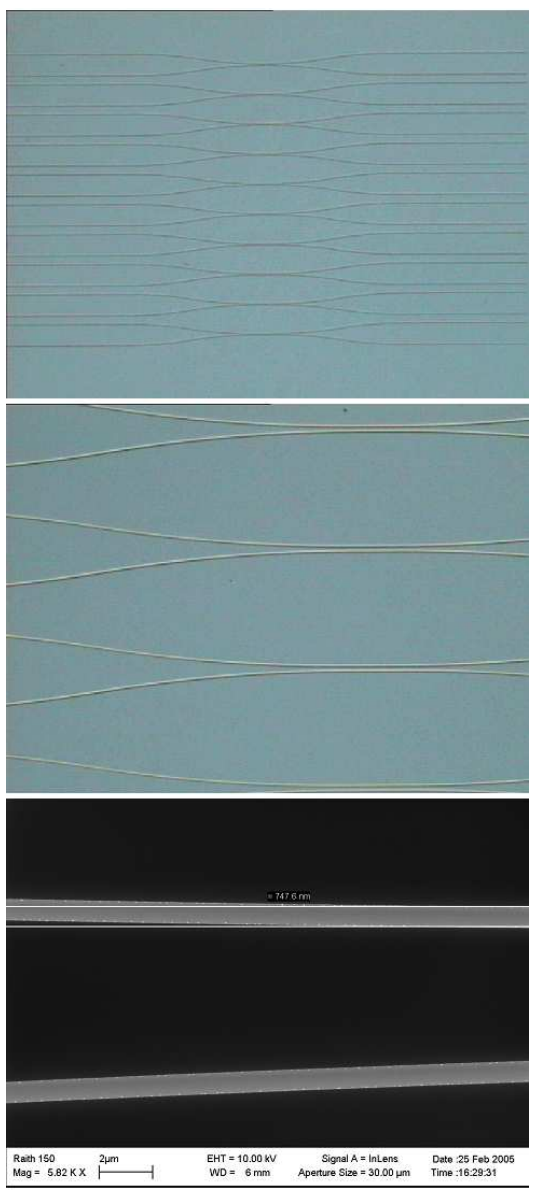

Fig. 3. Stitching-free optical waveguide couplers (increased magnification from top to bottom). M. Kahl, Raith GmbH. 
the range of $10 \mathrm{~nm}$. For optical waveguides, which could be many centimetres long, this causes a problem. Each boundary interface introduces a small loss due to the imperfection of the stitching. But many interfaces sum up to a big insertion loss. A special exposure mode with a fixed beam, but a fixed beam moving stage (FBMS) addresses this issue [11]. In this mode the waveguide is written in one continuous exposure path. The stage moves on a defined trajectory with a constant velocity while the electron beam does not change position. Figure 3 shows a series of optical waveguide couplers as an example. In this case the length of the waveguides is $2.5 \mathrm{~mm}$ (top image) and each waveguide has a width of $700 \mathrm{~nm}$. Each waveguide is written without any stitching error in one continuous path using FBMS.

\section{References}

[1] F. Robin, Electron-Beam Lithography Applications at ETH Zurich, Raith application note. Raith $\mathrm{GmbH}$, 2006.

[2] M.A. McCord, M.J. Rooks, in: Handbook of Microlithography, Micromachining, and Microfabrication, Vol. 1, Ed. P. Rai-Choudhury, 1997, p. 139.
[3] E.A. Shields, F. Williamson, J.R. Leger, J. Vac. Sci. Technol. B 21, 1453 (2003).

[4] G. Piaszenski, U. Barth, A. Rudzinski, A. Rampe, A. Fuchs, M. Bender, U. Plachetka, Microelectron. Eng. 84, 945 (2007).

[5] Technical description of the Raith150TWO instrument, Raith Germany.

[6] "Understanding of Hydrogen Silsesquioxane Electron Resist for Sub-5 nm-Half-Pitch Lithography", K.W. Joel Yang (MIT), oral presentation at EIBPN Conference, 2009.

[7] J.K.W. Yang, K.K. Berggren, J. Vac. Sci. Technol. B 25, 2025 (2007).

[8] S. Bauerdick, A. Linden, C. Stampfer, T. Helbling, C. Hierold, J. Vac. Sci. Technol. B 24, 3144 (2006).

[9] F. Robin, S. Costea, G. Stark, R. Wüest, P. Strasser, H. Jäckel, "Accurate Proximity-Effect Correction of Nanoscale Structures with NanoPECS", Raith application note, Raith GmbH.

[10] L.J. Martinez, I. Prieto, B. Alen, P.A. Postigo, J. Vac. Sci. Technol. B 27, 1801 (2009).

[11] M. Kahl, "Zero Stitching Error Using Fixed Beam Moving Stage (FBMS) Mode", Raith application note, Raith GmbH, 2005. 